\title{
PROCESS MONITORING, CONTROLLING AND LOAD MANAGEMENT SYSTEM IN AN INDUCTION MOTOR
}

\author{
R. Sujitha ${ }^{1}$, M. Dharmalingam ${ }^{2}$ \\ ${ }^{1}$ PG Scholar, Dept of ECE, Kongunadu College of Engg and Technology \\ ${ }^{2}$ Associate Professor, Dept of ECE, Kongunadu College of Engg and Technology
}

\begin{abstract}
A networked wireless industrial load management along with the process monitoring and controlling system in an induction motor based on wireless sensor networks An embedded sys-tem is employed for acquiring electrical signals from the motor and then performing local processing for voltage, current, temperature and speed estimation after transmitting to a monitoring unit through an ZigBee technology these parameters can be controlled using the labview software.
\end{abstract}

Keywords: embedded systems, induction motors, torque measurement, wireless sensor networks (WSNs), labview. $* * *$

\section{INTRODUCTION}

In associate industrial setting, mechanical systems driven by electrical motors are utilized in most production processes, accounting for quite common fraction of trade electricity consumption. Concerning the sort of motors typically utilized, regarding ninetieth are three-phase ac induction based mostly, in the main as a result of its value effectiveness and mechanical hardiness.

Voltage, current and speed are the most parameters for production machine's. In many trade sectors, these measurements could result in associate breakdown, that makes their observation essential so as to avoid disasters in vital production processes (e.g., oil and gas, mining, and sugar and alcohol industries). The calculable parameters from the motor's electrical signals makes the system less invasive, however it's less correct in comparison to direct measure systems.

Besides the high value, the wired approach offers very little flexibility, creating the network preparation and maintenance a tougher method. so the wireless networks gift variety of benefits compared to wired networks. additionally thereto, wireless device networks (WSNs) offer self-organization and native process capability. Therefore, these networks seem as a versatile and cheap answer for building industrial observation and management systems.

This paper presents associate embedded system for determinative voltage, current associated speed of an induction motor by using WSNs technology like ZIGBEE. Results ar then sent to a base unit for period observation and controlled victimisation the LABVIEW software package.

\section{BACKGROUND}

\subsection{Torque and Speed}

Torque, moment or moment of force, is the tendency of a force to rotate an object about an axis or pivot. Just as a force is a push or a pull, a torque can be thought of as a twist to an object. The relationship between speed and torque can be



Fig: 1 Relationship between torque and speed

\subsection{Industrial WSN}

WSNs are formed by devices equipped with sensors and are capable of communicating via radio frequency. These sensors can produce responses to changes in physical conditions such as temperature, humidity, or magnetic field. Specific types of WSNs, such as for industrial monitoring, have unique characteristics and specific application requirements. Therefore, the deployment of WSNs must necessarily involve considerations of the targeted application.

The IEEE 802.15.4 standard is well suited for WSN applications. It provides wireless communication with low power consumption and low cost, for monitoring and control 
applications that do not require high data transmission rate. There are some protocols that implement the network layer over the IEEE 802.15.4 standard, such as Zigbee and MiWi. The standard defines three frequency bands: $868 \mathrm{MHz}, 915$ $\mathrm{MHz}$, and $2.4 \mathrm{GHz}$. In this study, we have considered only the 2.4-GHz band.

Since there is no protection against interference of concurrent users, it is necessary to develop efficient coexistence technologies, providing a good operation of unlicensed band systems. Therefore, it is necessary to develop a new approach for wireless communication systems design, which should include spectrum occupation measurements, modeling of interference and coexistence, and performance evaluations.

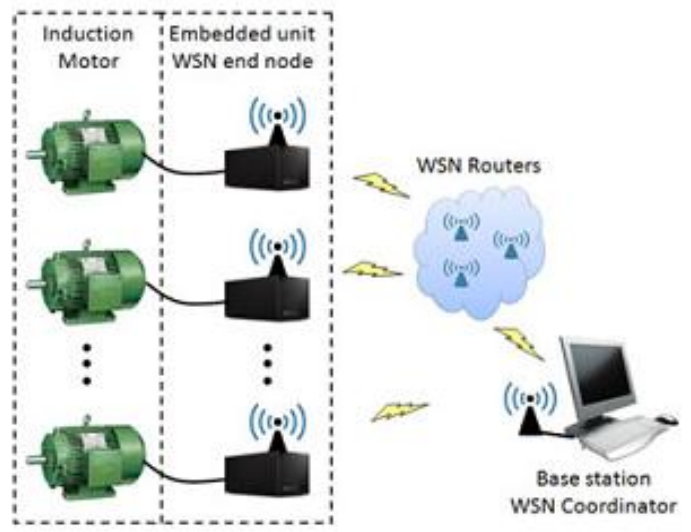

Fig: 2 embedded systems integrated into WSN

\section{SYSTEM ANALYSIS}

Monitoring system through wireless network and the Current status of system are updated to in charge personal. Prevention are taken before fault occurrence also the "LAB VIEW" software for monitoring .Data bases are created using software for analysis purpose

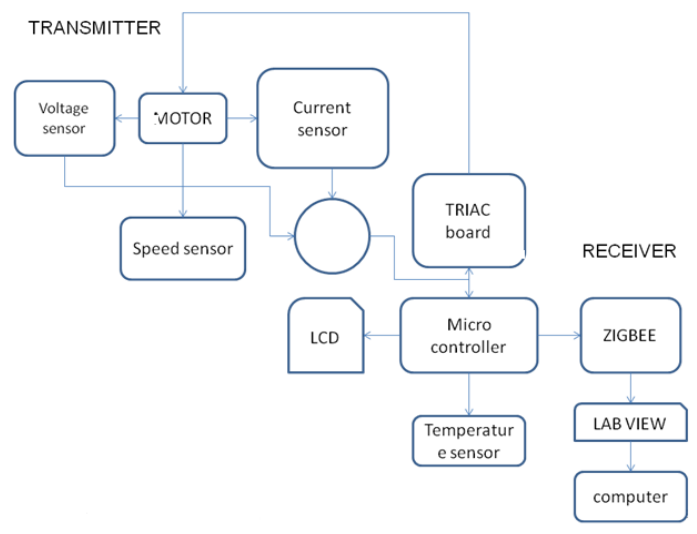

Fig: 3 block diagram

\section{HARDWARE DISCRIPTION}

\subsection{Voltage Divider}

A voltage divider is a simple circuit consisting of two resistors that has the useful property of changing a higher voltage (Vin) into a lower one (Vout). It does this by dividing the input voltage by a ratio determined by the values of two resistors (R1 and R2):

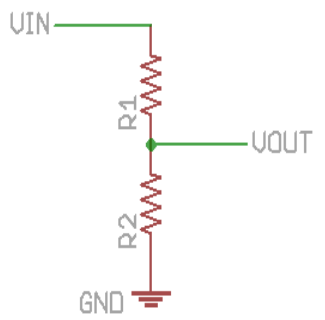

Fig 4 Voltage divider

This circuit is best for low-current applications like sensor and data lines. If you draw too much current through Vout it will affect the output voltage. Therefore this shouldn't be used for high-current applications like power supplies (voltage regulators are a much better option). To pick resistors, use the following equation:

$$
V_{\text {out }}=V_{\text {in }} \frac{R_{2}}{\left(R_{1}+R_{2}\right)}
$$

Because the output voltage depends solely on the ratio of $\mathrm{R} 1$ to $\mathrm{R} 2$, you could use a number of different $\mathrm{R}$ values to get the same output (for example, if $\mathrm{R} 1=\mathrm{R} 2$, the output will always

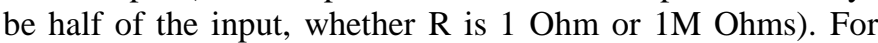
most of our purposes, the total resistance $(\mathrm{R} 1+\mathrm{R} 2)$ should be between 1k Ohms and 10k Ohms.
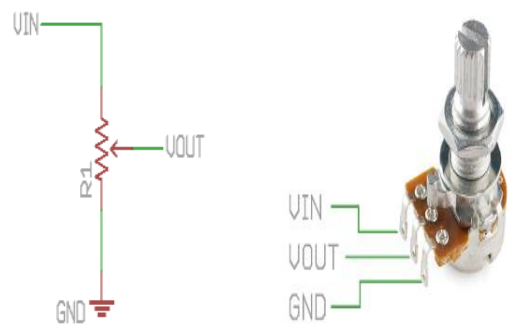

Fig 5

Inside a potentiometer is one big resistor with a moving connection (called a "wiper") that can move from one end of the resistor to the other. 
When you turn the knob, the ratio between the two resistors will change, smoothly varying the output voltage between Vin and GND.

\subsection{Speed Sensor}

Measurement of speed is of great significance in variety of application involving some form of motion. While moving in a car/ train or flying in an aircraft or sailing in a ship, we are often interested to know how fast we are moving. In engine control systems, it is imperative to know the speed of moving gears (camshaft/ crankshaft).

\subsection{Pin Functions}

There are pins along one side of the small printed board used for connection to the microcontroller. There are total of 14 pins marked with numbers (16 in case the background light is built in).

\subsection{Temperature Sensor}

National Semiconductor's LM35 IC has been used for sensing the temperature. It is an integrated circuit sensor that can be used to measure temperature with an electrical output proportional to the temperature. The temperature can be measured more accurately with it than using a thermistor. The sensor circuitry is sealed and not subject to oxidation, etc.

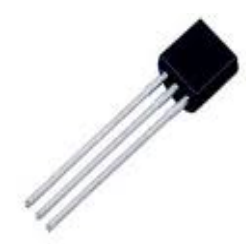

Fig: 6 Temperature sensor

\subsection{Wireless Sensor Network}

The technology defined by the zigbee specification is intended to be simpler and less expensive than other wpans, such as bluetooth. Zigbee is targeted at radio-frequency (rf) applications that require a low data rate, long battery life, and secure networking.

ZigBee is the name given to a specific suite of high level communication protocols using low power digital radios, based on the IEEE 802.15.4 standard for Wireless Personal Area Networks (WPANs).

The following diagram relates a number of wireless technologies used in WPANs, WLANs (Wireless Local Area Networks,) WMANs (Wireless Metropolitan Areas) and WWNAs (Wireless Wide Area Networks.) The speeds shown are guides only. WWANs are dominated by mobile phone (cell phone) technologies, known as 2G, 3G and, forthcoming, $4 \mathrm{G}$.



Fig: 7 Zigbee transceiver

WPANs cover a radius of about $10 \mathrm{~m}$ around a person or object. The core aim is to design systems offering low cost, low power, and compact size. The IEEE 802.15 working group has defined three classes of WPANs, differentiated by data rate, power requirements and level of performance.

Table 1: Specification of Zigbee

\begin{tabular}{|c|c|c|c|}
\hline Frequency & ISM $2.4-2.484 \mathrm{GHz}$ & ISM 2.4-2.484 GHz & ISM $2.4-2.484 \mathrm{GHz}$ \\
\hline Nominal Transmit Power & $1 \mathrm{~mW}(+0 \mathrm{dBm})$ & $158 \mathrm{~mW}(+22 \mathrm{dBm})$ & $1 \mathrm{~W}(+30 \mathrm{dBm})$ \\
\hline RF Data Rate & $250 \mathrm{Kbps}$ & $250 \mathrm{Kbps}$ & $250 \mathrm{Kbps}$ \\
\hline Receiver Sensitivity & $.92 \mathrm{dBm}$ & $-103 \mathrm{dBm}$ & $-104 \mathrm{dBm}$ \\
\hline Serial Data Rate & Up to 115200 baud & Up to 115200 baud & Up 10115200 baud \\
\hline Operating Temperature & $401085^{\circ} \mathrm{C}$ & -40 to $85^{\circ} \mathrm{C}$ & -40 to $85^{\circ} \mathrm{C}$ \\
\hline Antenna Connector & MMCX & MMCX & MMCX \\
\hline $\begin{array}{l}\text { On-board Antenna } \\
\text { Options }\end{array}$ & $\begin{array}{l}\text { Chip Antenna } \\
\text { Wire Antenna }\end{array}$ & $\begin{array}{l}\text { Chip Antenna } \\
\text { Wire Antenna }\end{array}$ & NA \\
\hline
\end{tabular}

\section{SYSTEM IMPLEMENTATION}

\subsection{Labview}

LabVIEW (short for Laboratory Virtual Instrument Engineering Workbench) is a system-design platform and development environment for a visual programming language the programming language used in LabVIEW, also referred to as $G$, is a dataflow programming language. Execution is determined by the structure of a graphical block diagram (the LabVIEW-source code) on which the programmer connects different function-nodes by drawing wires.

\subsection{Code Compilation}

In terms of performance, LabVIEW includes a compiler that produces native code for the CPU platform. The graphical code is translated into executable machine code by interpreting the syntax and by compilation. The LabVIEW syntax is strictly enforced during the editing process and compiled into the executable machine code when requested to run or upon saving. In the latter case, the executable and the source code are merged into a single file. 


\subsection{Large Libraries}

Many libraries with a large number of functions for data acquisition, signal generation, mathematics, statistics, signal conditioning, analysis, etc., along with numerous graphical interface elements are provided in several LabVIEW package options. The number of advanced mathematic blocks for functions such as integration, filters, and other specialized capabilities usually associated with data capture from hardware sensors is immense.

\subsection{Code Re-Use}

The fully modular character of LabVIEW code allows code reuse without modifications: as long as the data types of input and output are consistent, two sub VIs are interchangeable. The LabVIEW Professional Development System allows creating stand-alone executables and the resultant executable can be distributed an unlimited number of times. The run-time engine and its libraries can be provided freely along with the executable.

\section{RESULTS}

An embedded system integrated into a WSN for monitoring and controlling an induction motors. We used the LABVIEW software for controlling the voltage current and speed. The calculations for estimating the targeted values are done locally and then transmitted to a monitoring base unit through an ZIGBEE.

Experimental tests were also performed to analyze the voltage, current and speed values of an single phase induction motor.

\subsection{Blocks in Labview}



Fig 8 Serial communication block with identifier

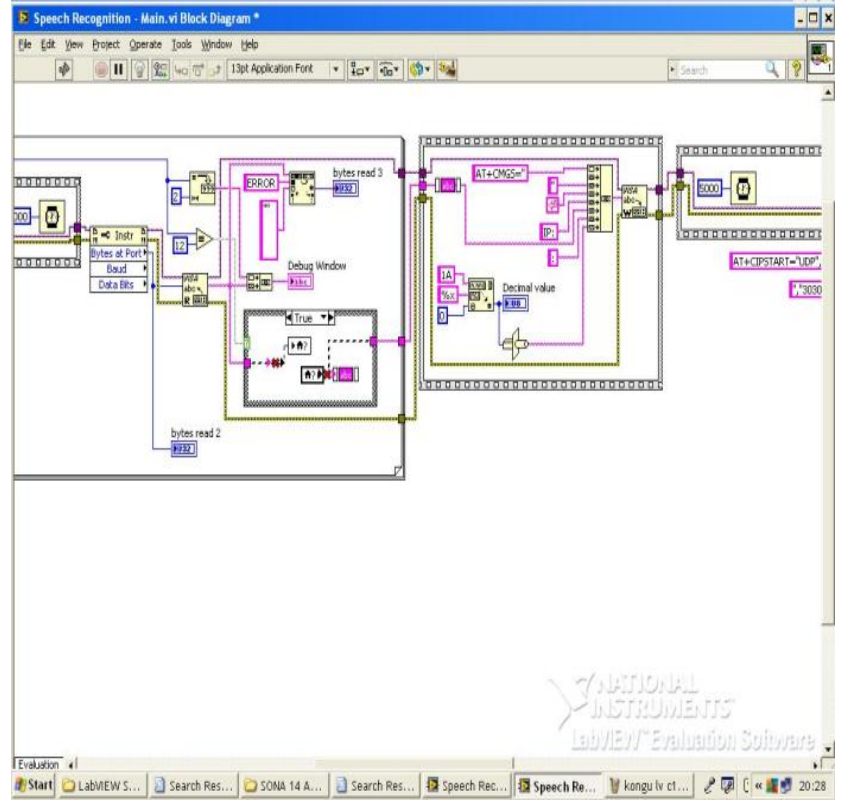

Fig 9 Microcontroller block

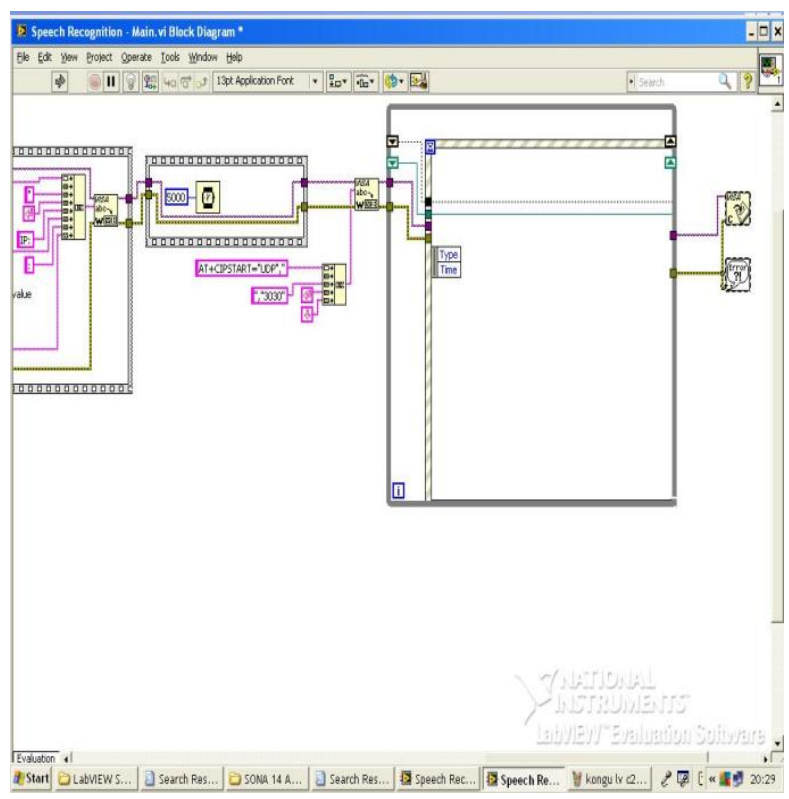

Fig 10 Display blocks

\section{CONCLUSIONS}

The induction motor parameters such as voltage, current and speed is monitored and controlled using the software along with the experimental results.

For the controlling purpose of an induction motor in hardware ,triac board is used and in software labview is used. Thus the verification can be done both in hardware as well as software and the parametric waveforms is obtained. 


\section{REFERENCES}

[1] R.Hanitsch (2002)'Energy Efficient Electric Motors' in Proc.

[2] K. Kim and A. G. Parlos, "Induction motor fault diagnosis based on neu-ropredictors and wavelet signal processing," IEEE/ASME Trans. Mechatronics, vol. 7, no. 2, pp. 201-219, Jun..

[3] C. Lima-Filho, F. A. Belo, and R. D. Gomes, "Tests prove, self-powered, wireless, pump torquemeter," Oil Gas J., vol. 106, no. 46, pp. 43-48, 2008.

[4] R. B. Reich, "Rotary transformer," U.S. Patent 4412 198, Oct. 25, 1983.

[5] W.F.Buchele, "Strain-gauge brushless torque-meter," U.S. Patent 3881 347, May 6, 1975.

[6] Z. Meng and B. Liu, "Research on torque real time monitoring system of rotary machine," Chin. J. Sci. Instrum., vol. 26, pp. 38-39, 2005.

[7] C. Lima-Filho, F. A. Belo, J. L. A. Santos, and E. G. Anjos, "Exper-imental and theoretical study of a telemetric dynamic torque meter," J. Brazilian Soc. Mech. Sci. Eng., vol. 32, pp.241-249, 2010.

[8] J.S. Hsu and A. M. A. Amin, "Torque calculations of current-source induction machines," IEEE Trans.., vol. 37, no. 1, pp. 34-40, Feb. 1990.

[9] J. S. Hsu, "Capacitor effects on induction motors fed by quasi rectangular current sources," IEEE Trans. Energy Convers., vol. 7, no. 3, pp. 509-516, Sep. 1992.

[10] T. H. Lee, T.S. Low, K.J. Tseng, and H. K.Lim, “An intelligent indirect dynamic torque sensor for permanent magnet brushless DC drives," IEEE Trans. Ind. Electron., vol. 41, no 2, pp. 191-200,1994 